\title{
Tantalizing dilaton tests from a near-conformal EFT
}

\author{
Zoltan Fodor \\ University of Wuppertal, Department of Physics, Wuppertal D-42097, Germany \\ Juelich Supercomputing Center, Forschungszentrum Juelich, Juelich D-52425, Germany \\ Eotvos University, Pazmany Peter setany 1, 1117 Budapest, Hungary \\ fodor@bodri.elte.hu
}

Kieran Holland

University of the Pacific, 3601 Pacific Ave, Stockton CA 95211, USA

Albert Einstein Center for Fundamental Physics, Bern University, Bern, Switzerland

khollandepacific.edu

Julius Kuti*

University of California, San Diego, 9500 Gilman Drive, La Jolla, CA 92093, USA

jkuti@ucsd.edu

\section{Chik Him Wong ${ }^{\dagger}$}

University of Wuppertal, Department of Physics, Wuppertal D-42097, Germany

cwongeuni-wuppertal.de

The dilaton low-energy effective field theory (EFT) of an emergent light scalar is probed in the paradigm of strongly coupled near-conformal gauge theories. These studies are motivated by models which exhibit small $\beta$-functions near the conformal window $(\mathrm{CW})$, perhaps with slow scale-dependent walking and a light scalar with $0^{++}$quantum numbers. We report our results from the hypothesis of a dilaton inspired EFT analysis with two massless fermions in the twoindex symmetric (sextet) representation of the SU(3) color gauge group. With important caveats in our conclusions, conformal symmetry breaking entangled with chiral symmetry breaking would drive the near-conformal infrared behavior of the theory predicting characteristic dilaton signatures of the light scalar from broken scale invariance when probed on relevant scales of fermion mass deformations. From a recently reasoned choice of the dilaton potential in the EFT description [1] we find an unexpectedly light dilaton mass in the chiral limit at $m_{d} / f_{\pi}=1.56(28)$, set in units of the pion decay constant $f_{\pi}$. Subject to further statistical and systematic tests of continued post-conference analysis, this result is significantly lower than our earlier estimates from less controlled extrapolations of the light scalar (the $\sigma$-particle) to the massless fermion limit of chiral perturbation theory. We also discuss important distinctions between the dilaton EFT analysis and the linear $\sigma$-model without dilaton signatures. For comparative reasons, we comment on dilaton tests from recent work with fermions in the fundamental representation with $n_{f}=8$ flavors.

The 36th Annual International Symposium on Lattice Field Theory - LATTICE2018

22-28 July, 2018

Michigan State University, East Lansing, Michigan, USA.

\footnotetext{
* Speaker.

† Speaker.
} 


\section{Introduction and overview}

Tantalus, a king of ancient Phrygia in Greek mythology, made the mistake of gravely offending the gods. As a punishment, once dead the king was forced to stand in a pool of water, with fruit hanging just over his head. The water would recede every time the king tried to take a sip, and the fruit would lift away every time he reached to take a bite.

New results are reported from the dilaton effective field theory analysis of a very light scalar with $0^{++}$quantum numbers in lattice simulations of a strongly coupled gauge theory, defined in the two-index symmetric fermion representation of the SU(3) color gauge group (one flavor doublet of massless fermions with sextet color). The sextet model thus defined plays a prominent role in the near-conformal gauge theory paradigm, perhaps with BSM implications.

In earlier work we discovered the light $0^{++}$scalar as one of the most significant theoretical and practical consequences of near-conformal infrared behavior in the sextet theory [2-6], radically different from the heavy $\sigma$-particle of gauge theories far from the conformal window and modeled like QCD. We investigate the hypothesis of a dilaton inspired EFT in the sextet model with important caveats in our conclusions. Accordingly, conformal symmetry breaking would be entangled with chiral symmetry breaking $(\chi S B)$ driving near-conformal infrared behavior and predicting characteristic dilaton signatures of the light scalar from broken scale invariance when probed on relevant scales of fermion mass deformations. We find an unexpectedly light dilaton mass in the chiral limit at $m_{d} / f_{\pi}=1.56(28)$, set in units of the pion decay constant $f_{\pi}$ from a recently reasoned choice of the dilaton potential in the Lagrangian of the EFT [1]. Subject to further statistical and systematic tests of continued post-conference analysis, this result is significantly lower than our earlier estimates for the $\sigma$-particle from less controlled extrapolations to the massless fermion limit of chiral perturbation theory without characteristic dilaton features.

Two models are identified in Section 2 with significantly smaller step $\beta$-functions than QCD and perhaps with slowly walking scale dependence correlated with dilaton signatures of emergent light scalars. Before pivoting to the dilaton analysis in Section 4 we present first in Section 3 the unresolved challenges of the standard $\chi P T$ analysis and its extensions to the linear $\sigma$-model. We also discuss important differences of the sextet dilaton analysis from chiral perturbation theory $(\chi P T)$ and from general extensions of the linear $\sigma$-model without dilaton signatures. In Section 4 the dilaton EFT is discussed and important predictions are reviewed for hypothesis testing. Results are reported from the current status of the sextet dilaton analysis. Based on the recently published data of the LSD collaboration [7] some of our own comparative $n_{f}=8$ dilaton analysis is also reported. New ideas and simulations are briefly presented in Section 5 for reaching much reduced fermion mass scales in the $\varepsilon$-regime of the dilaton EFT. We conclude in Section 6 with some cautionary remarks and caveats for the outlook.

\section{Near-conformal $\beta$-functions and the light $0^{++}$scalar}

The step $\beta$-function of the renormalized gauge coupling is shown from lattice studies in Fig. 1 for two different fermion representations of strongly coupled gauge theories with SU(3) gauge group [6]. Five $\beta$-functions are shown and three of them may exhibit dilaton-like features of their light scalar. In the fundamental representation, the $n_{f}=4$ model in Fig. 1 is like QCD with four massless fermions. It has the largest $\beta$-function [8] and a $\sigma$-particle with a heavier mass, $m_{\sigma} / F \sim 6$, set by the scale of the Goldstone decay constant $F$ in the massless fermion limit of $\chi S B$ [7]. (For convenience, we will change the notation to $F \equiv f_{\pi}$ in all other sections.) At increased flavor 


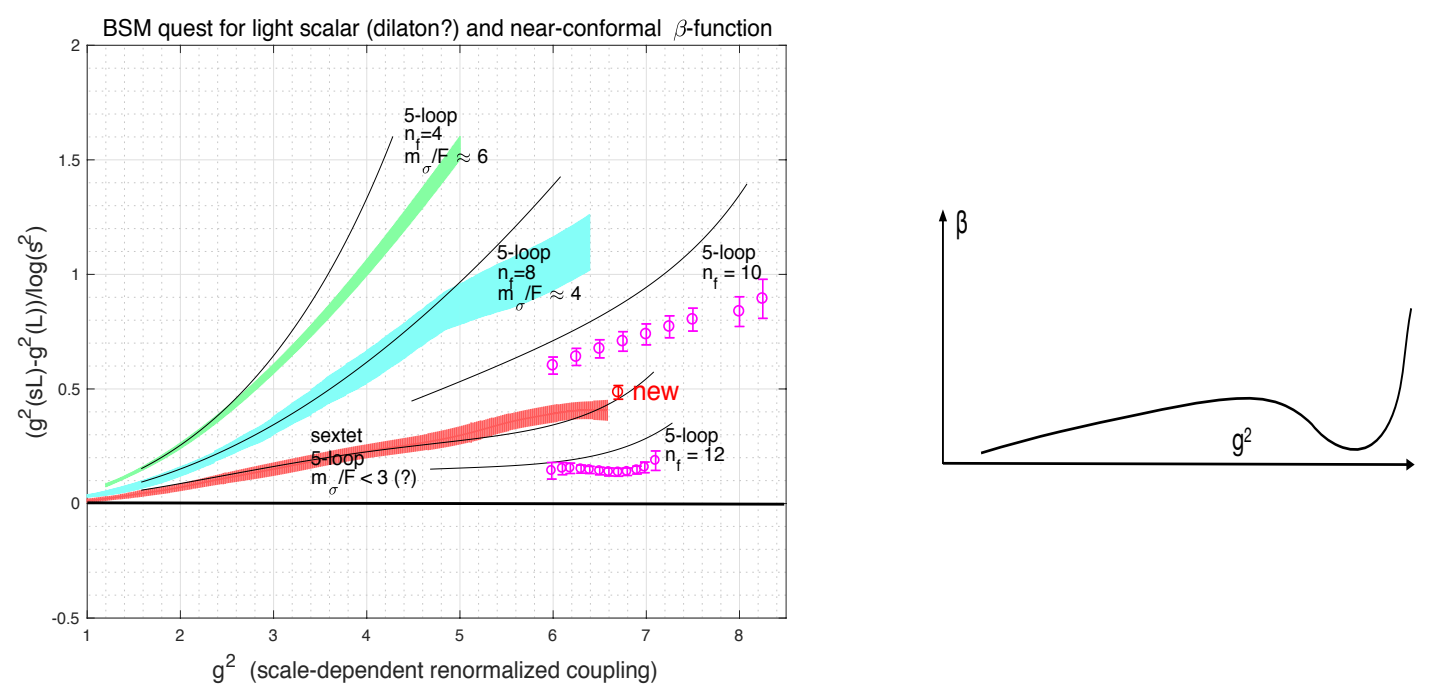

Figure 1: The five $\beta$-functions of the models surveyed in this section are shown on the left panel. The correlation with the emergent light scalars in the $n_{f}=8$ model and the sextet model are discussed in the text. The right panel is the cartoon version of a walking $\beta$-function, replaced by small and flat $\beta$-functions in realistic near-conformal models.

number $n_{f}=8$ the $\beta$-function becomes reduced and noticeably more flat compared to QCD [9] and exhibiting a scalar mass with significantly reduced value $[7,10,11]$. For the observed range of fermion mass deformations at $n_{f}=8$, the scalar mass and the pion mass are tracking each other. This is quite distinct from QCD, with motivation for the $n_{f}=8$ dilaton analysis in [12,13]. At $n_{f}=10$ the further increase of the flavor number leads to further reduction of the $\beta$-function $[14,15]$ with some unresolved controversy. An infrared fixed point was reported in [16] with vanishing $\beta$ function at $g^{2} \sim 7$ and reconsidered in follow-up work with improved systematics which left the conformal or near-conformal behavior of the $\beta$-function unresolved at strong gauge couplings [17]. The model with the largest flavor number in the fundamental representation at $n_{f}=12$ is shown in Fig. 1 with the smallest $\beta$-function exhibiting very flat dependence at strong coupling $[6,14]$. We will return in the future to the interesting challenge this model presents very close to the $\mathrm{CW}$, perhaps near-conformal and walking but with controversies from recent lattice work $[18,19]$ and from a new conference contribution [20] suggesting conformal behavior.

The sextet $\beta$-function in Fig. 1 is from [21] with the $m_{\sigma} / F$ ratio taken from [4]. The point marked as new was our Lattice 2017 conference contribution [22], bridging the volume dependent step $\beta$-function and the scale dependent $\beta$-function of the p-regime in the infinite volume limit. We have now a set of new gauge ensembles in the sextet model to extend the small and flat step $\beta$-function of Fig. 1 toward stronger gauge couplings. The mark (?) next to the sextet model ratio $m_{\sigma} / F<3$ is our indicator that the final ratio in the chiral limit requires further analysis, with this report contributing new results.

The above brief survey presents motivations for near-conformal tests of the $n_{f}=8$ model and the $n_{f}=2$ sextet model for dilaton signatures. The right panel of Fig. 1 is the cartoon version of a walking $\beta$-function, presumably replaced by small and flat $\beta$-functions in realistic near-conformal models. Although the unrealistic cartoon shape of the $\beta$-function might be sufficient for dilaton analysis when the conformal limit is approached in some parametric expansion, we remain uncon- 
vinced that it is a required feature of walking. Recent 5-loop results are only plotted in Fig. 1 to indicate the state of the art in the perturbative loop expansion [23], perhaps for future theoretical analysis of walking, based on speculations for a pair of complex conformal fixed points of walking non-perturbative $\beta$-functions below the $\mathrm{CW}$. It did not escape our attention that investigations of this scenario should also include the $n_{f}=12$ model.

\section{Challenges of the sextet $\chi$ PT analysis and its linear $\sigma$-model extensions}

\subsection{Early discovery of the light scalar and the associated particle spectrum}

The light $0^{++}$scalar in the two-index symmetric (sextet) fermion representation of the SU(3) color gauge group was reported first at Lattice 2013 in [2,3] as shown in the left panel of Fig. 2. We
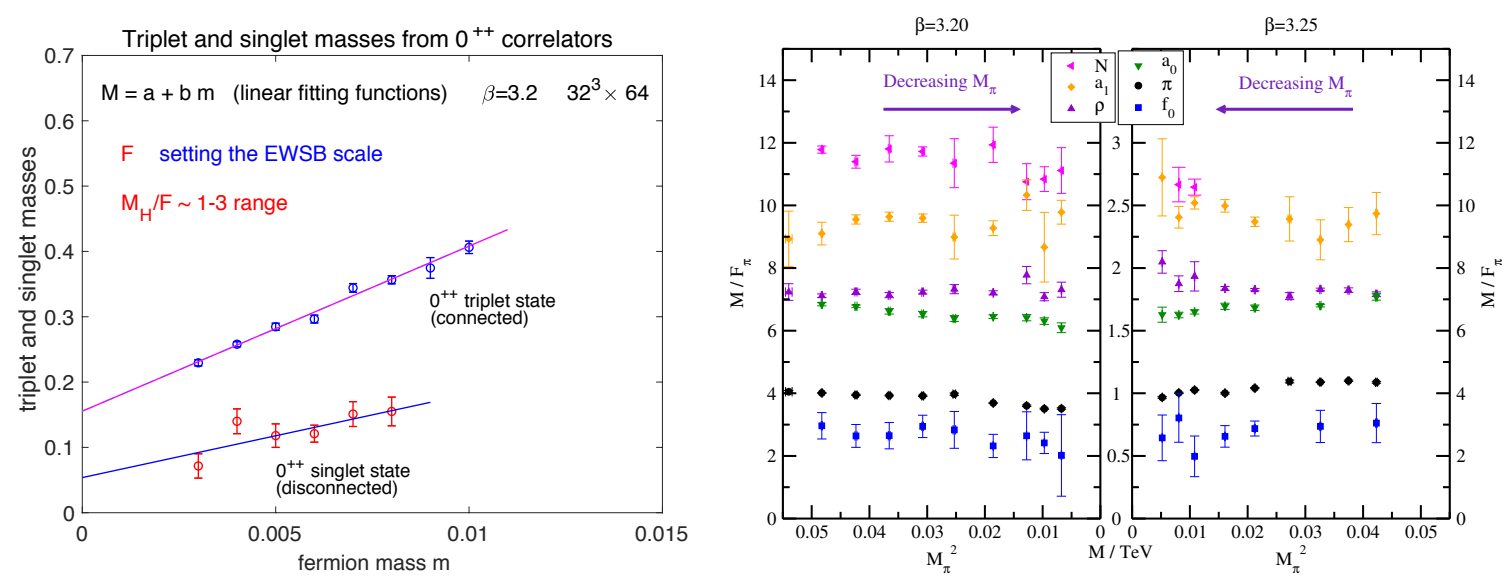

Figure 2: (left) The first result on the light sextet scalar from [2,3]; (right) The status of hadron spectroscopy in the sextet model is shown as reported in [4] including $M_{\pi}, F_{\pi}$ and the scalar mass $M_{d}\left(M_{d} \equiv M_{H}\left(f_{0}\right)\right.$ in earlier notation). The $M_{\pi}, F_{\pi}, M_{d}$ data set of the analysis has been updated and refined since using new lattice ensembles in large volumes. Input data $M_{d}$, as used in some parts of the dilaton analysis, comes from the report in [4] as graphically represented on the right panel of the figure.

have our $M_{\pi}, F_{\pi}, M_{d}$ sextet data set $\left(M_{d} \equiv M_{H}\left(f_{0}\right)\right.$ in earlier notation) from a very large number of gauge ensembles at three lattice spacings in a range of fermion masses, $m=0.0010-0.0080$, with lattices sizes from $32^{3} \times 64$ to $64^{3} \times 96$. The finite size analysis of the data set was presented in [6]. We use infinite volume extrapolations of $M_{\pi}, F_{\pi}$ data sets at fixed bare gauge coupling $\beta=6 / \mathrm{g}^{2}$, with $\beta=3.20$ at each of the lowest five input fermion masses applied to the analysis. The $M_{d}$ input is always taken from the largest volume of the gauge ensembles at each input fermion mass.

\subsection{Pivot to dilaton EFT from $\chi \mathrm{PT}$ and its linear sigma model extensions}

Motivated by the $S U(2)$ doublet of mass deformed Goldstone pions of the sextet model, we tested mass deformed chiral perturbation theory $(\chi P T)$ when applied to the above described sextet data for $M_{\pi}$ and $F_{\pi}$. As shown in Fig. 3, the logarithmic form of NLO one-loop $\chi P T$ can be separately fitted to $M_{\pi}$ with the three parameters $B_{\pi}, f_{\pi}, \Lambda_{3}$ and good $\chi^{2}$ for the one-loop chiral Lagrangian. Similarly, $F_{\pi}$ fits well with a separate set of three parameters $B_{\pi}, f_{\pi}, \Lambda_{4}$ and good $\chi^{2}$. 
However, NLO $\chi P T$ fails for simultaneous fits of the $M_{\pi}, F_{\pi}$ input set because the two pairs $B, f_{\pi}$ of low-energy parameters of the $\chi P T$ Lagrangian are inconsistent in separate fits of $M_{\pi}$ and $F_{\pi}$.

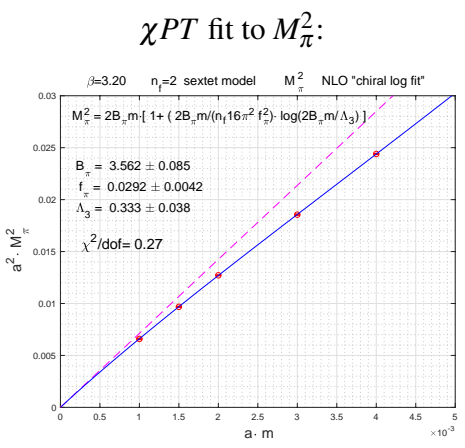

Conformal fit to $M_{\pi}$ :

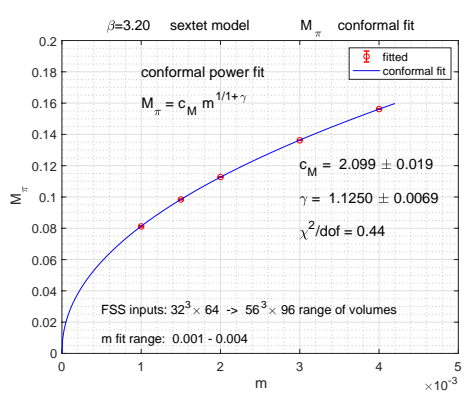

$\chi P T$ fit to $F_{\pi}$ :

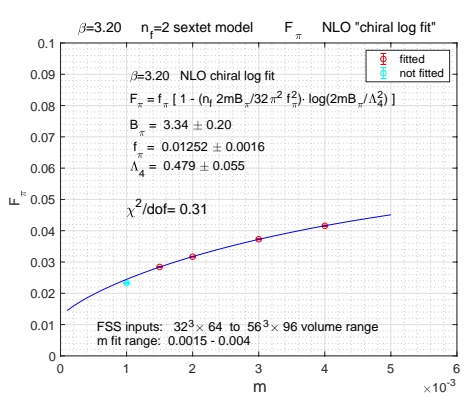

Conformal fit to $F_{\pi}$ :

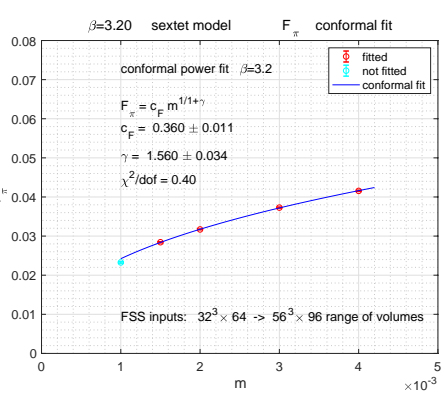

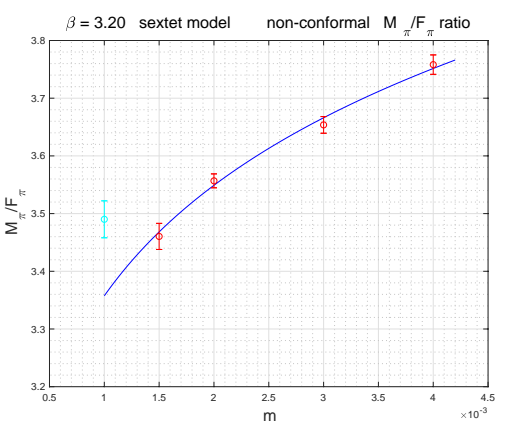

Failed conformal fit with large variation of the $M_{\pi} / F_{\pi}$ ratio in the fitted fermion mass range.

Figure 3: Chiral and conformal fits of the $M_{\pi}, F_{\pi}$ parameters in the sextet model. The lowest $m$ is not fitted in $F_{\pi}$ from incomplete finite size scaling analysis.

Forcing conformal behavior on the sextet model is not the answer. The results in Fig. 3 from forced conformal fits to $M_{\pi}$ and $F_{\pi}$ show unacceptable results in the mass range of our analysis with significant variation of the $M_{\pi} / F_{\pi}$ ratio and inconsistent conformal $\gamma$ exponents, statistically rejected on any reasonable level of confidence. We remain puzzled and unconvinced by recent claims of observing conformal behavior in the model [24].

As a possible remedy to the failures of $\chi P T$, we had been experimenting in the past with rooted staggered $\chi P T$ to include cutoff effects with taste breaking from the staggered pion spectrum. The results at $\beta=3.20$ were reported in [6] but applying the same analysis at $\beta=3.25$ new problems emerged. The plausible interpretation of the failing $\chi P T$ analysis is the light scalar closely tracking the mass-deformed pion spectrum as shown in Fig. 2. Pion dynamics remains closely coupled to $0^{++}$scalar dynamics, violating the basic premise of $\chi P T$.

In the sextet model with $S U(2) \times S U(2)$ flavor group the linear $\sigma$-model would be a natural candidate to extended $\chi P T$ to the coupling of the Goldstone triplet to the $\sigma$-particle in the $m_{\sigma} / F<3$ mass range with broken $S U(2) \times S U(2) \sim O(4)$ symmetry. This would match the construction of the standard model Higgs sector at a higher $m_{\sigma}$ mass in the presence of fermion mass deformations, before extrapolation to the chiral limit is taken. However, the linear $\sigma$-model with broken $S U(2) \times S U(2) \sim O(4)$ symmetry is not applicable in the current range of fermion mass deformations where existing data with $m_{\sigma} \sim m_{\pi}$ do not comply with the condition $m_{\sigma}^{2} \geq 3 m_{\pi}^{2}$ derived from the tree-level Lagrangian of the linear $\sigma$-model [25]. Much lower fermion mass deformations would be needed to probe the linear $\sigma$-model regime. Following earlier work of Soto [26], we were experimenting with extensions of the linear $\sigma$-model to find relevant additional terms for the anal- 
ysis, similar to Eq.(4.1) with the choice $V_{\sigma}$ from Eq.(4.2b) but with preset value of the $y$ exponent at $y=2$ in the mass term of the Lagrangian, making the dilaton EFT practically indistinguishable from the generalized linear $\sigma$-model. We performed one-loop calculations at $y=2$ and made tree level fits to our sextet lattice ensembles without satisfactory results ( in the meantime, complete one-loop calculations appeared in [27]). While working on loop correction in the analysis and the relaxation of the $y=2$ constraint to accommodate the more general dilaton analysis of the sextet model, the pioneering tree level $n_{f}=8$ dilaton analysis appeared [12] and the two groups were off to the dilaton races [12,14]. In this spirited competition we published the first sextet analysis [14]. A follow-up publication to [12] appeared [13] extending the $n_{f}=8$ analysis and including some limited sextet analysis based on plots from our earlier sextet publications without access to our exclusive and more recent data sets. In the next section we provide some technical details of our significantly extended dilaton analysis beyond what was published in [14] for the sextet model. For comments and comparative purposes, we also include our extended $n_{f}=8$ analysis based on recently published data from [7].

\section{Dilaton effective field theory of two candidates for near-conformal models}

\subsection{The EFT Lagrangian}

In Section 2 we presented evidence for the correlated trend between the reduced mass of the $0^{++}$singlet scalar and the reduced size of the $\beta$-function close to the lower edge of the CW suggesting near-conformal behavior in the sextet model. In the currently accessible range of fermion mass deformations, the mass of the light scalar is tracking closely the Goldstone boson (pion) multiplet from spontaneous chiral symmetry breaking $(\chi S B)$ of the underlying $S U\left(n_{f}\right) \times S U\left(n_{f}\right)$ flavor group, with $n_{f}=2$ in the sextet model. This characteristic behavior is captured in a recently investigated low-energy EFT $[1,12,13,28]$ to describe the light $\sigma$-particle with $0^{++}$quantum numbers, coupled to pion dynamics as a dilaton from broken scale invariance. The first application to the $n_{f}=8$ model was reported in [12,13]. After our first analysis of the sextet model in [6] we report here a broader scope of dilaton EFT tests in the sextet and $n_{f}=8$ models.

The minimal modification of the chiral Lagrangian with dilaton couplings leads to the EFT

$$
\mathscr{L}=\frac{1}{2} \partial_{\mu} \chi \partial_{\mu} \chi-V(\chi)+\frac{f_{\pi}^{2}}{4}\left(\frac{\chi}{f_{d}}\right)^{2} \operatorname{tr}\left[\partial_{\mu} \Sigma \partial_{\mu} \Sigma^{\dagger}\right]+\frac{m_{\pi}^{2} f_{\pi}^{2}}{4}\left(\frac{\chi}{f_{d}}\right)^{y} \operatorname{tr}\left[\Sigma+\Sigma^{\dagger}\right],
$$

where the notation $\chi=f_{d} \cdot e^{\sigma / f_{d}}$ is introduced for the connection between the dilaton field $\sigma(x)$ and the compensator field $\chi(x)$ which transforms as $\chi(x) \rightarrow \chi^{\prime}\left(x^{\prime}\right)=e^{\omega} \chi(x)$ under the shift $\sigma(x) \rightarrow$ $\sigma^{\prime}\left(x^{\prime}\right)=\sigma(x)+\omega \cdot f_{d}$ for scale transformations $x_{\mu} \rightarrow x_{\mu}^{\prime}=e^{-\omega} x_{\mu}$. The notation $f_{d}$ designates the minimum of the dilaton potential $V(\chi)$ in the chiral limit of vanishing fermion masses. In Eq. (4.1) of the EFT two different forms of the dilaton potential were chosen for our analysis,

$$
\begin{aligned}
& V(\chi) \rightarrow V_{d}(\chi)=\frac{m_{d}^{2}}{16 f_{d}^{2}} \chi^{4}\left(4 \ln \frac{\chi}{f_{d}}-1\right), \\
& V(\chi) \rightarrow V_{\sigma}(\chi)=\frac{m_{d}^{2}}{8 f_{d}^{2}}\left(\chi^{2}-f_{d}^{2}\right)^{2} .
\end{aligned}
$$


Recent theoretical motivation of Eq.(4.2a) originates from [1], based on a parametric expansion of $V(\chi)$ as the $\mathrm{CW}$ is approached. Eq.(4.2b) is a linear $\sigma$-model inspired dilaton in [12, 13,29]. We do not dwell on various aspects of the two choices in our first extended sextet tests. The primary focus is on comparing the two choices $V_{\sigma}, V_{d}$ and commenting on what was reported in earlier work $[12,13,30]$ and at the conference [31]. We will show that the choice of $V_{d}$, favored by the theory in [1], might become a game changer in the interpretation of the sextet model.

The Goldstone pions in Eq.(4.1) are described by the unitary matrix field $\Sigma=\exp \left[2 i \pi / f_{\pi}\right]$ where the pion field is represented as $\pi=\Sigma_{a} \pi^{a} T^{a}$ with $n_{f}^{2}-1$ generators of the $S U\left(n_{f}\right)$ flavor group. We keep the same notation as in $[12,13]$ for the parameters in Eqs. $(4.1,4.2 a, 4.2 b)$ for the convenience of easy comparison with our analysis. In this notation, the tree level pion mass would be $m_{\pi}^{2}=2 B_{\pi} m$ close to the chiral limit, with the dilaton decoupled from pion dynamics. The pion decay constant $f_{\pi}$ is defined in the chiral limit. The tree-level dilaton mass in the chiral limit of vanishing fermion mass is designated as $m_{d}$ and it is defined by the second derivative of the treelevel dilaton potential at its $\chi=f_{d}$ minimum as $V^{\prime \prime}\left(\chi=f_{d}\right)=m_{d}^{2}$. The dilaton mass at finite fermion mass deformations is designated by $M_{d}$.

The scale-dependent anomalous dimension of the chiral condensate, as $y=3-\gamma$ in Eq.(4.1), will require some more refined scale setting definition in walking theories and will not be addressed here. In the sextet model we have detailed information on the scale-dependent $\gamma$ which will be compared with the results emerging from the analysis of Eqs.(4.1,4.2a,4.2b).

The Lagrangian of the dilaton EFT in Eq.(4.1) has a long history which includes [29, 32-42] with further references.

\subsection{MCMC analysis of dilaton EFT predictions from $\mathrm{V}_{d}$ and $\mathrm{V}_{\sigma}$ potentials: sextet model}

In Markov Chain Monte Carlo (MCMC) based analysis of the implicit Maximum Likelihood (IML) procedure, the targeted five physical parameters $f_{\pi}, B_{\pi}, y, m_{d} / f_{\pi}, f_{d} / f_{\pi}$ are defined by treelevel application of the dilaton EFT, based on Eq.(4.1). For the choice of the dilaton potential $V_{d}(\chi)$ the physical parameters are subject to three non-linear constraints at each input fermion mass $m$ leading to twelve constraints with input at four different fermion masses in the IML procedure,

$$
\begin{aligned}
& M_{\pi}^{2} \cdot F_{\pi}^{2-y}-2 B_{\pi} \cdot f_{\pi}^{(2-y)} \cdot m=0, \\
& F_{\pi}^{(4-y)} \cdot \log \left(F_{\pi} / f_{\pi}\right)-y \cdot n_{f} f_{\pi}^{(6-y)} B_{\pi} \cdot m / m_{d}^{2} f_{d}^{2}=0, \\
& \left(F_{\pi}^{2} / M_{\pi}^{2}\right) \cdot\left(3 \log \left(F_{\pi} / f_{\pi}\right)+1\right)-\left(M_{d}^{2} / m_{d}^{2}\right) \cdot\left(f_{\pi}^{2} / M_{\pi}^{2}\right)-y(y-1) n_{f} f_{\pi}^{4} / 2 m_{d}^{2} f_{d}^{2}=0 .
\end{aligned}
$$

The general scaling relation of Eq.(4.3) is independent from the choice of the dilaton potential $[12,28]$. The dilaton potential $V_{d}$ leads to two added non-linear conditions, with Eq.(4.4) set by $V_{d}^{\prime}\left(\chi=F_{d}\right)$, and Eq.(4.5) set by $V_{d}^{\prime \prime}\left(\chi=F_{d}\right)$, as in [12]. With unchanged scaling relation from Eq.(4.3), two alternative equations are derived from $V_{\sigma}^{\prime}\left(\chi=F_{d}\right)$ and $V_{\sigma}^{\prime \prime}\left(\chi=F_{d}\right)$,

$$
\begin{aligned}
& F_{\pi}^{(4-y)} \cdot\left(1-f_{\pi}^{2} / F_{\pi}^{2}\right)-2 y \cdot n_{f} f_{\pi}^{(6-y)} B_{\pi} / m_{d}^{2} f_{d}^{2} \cdot m=0, \\
& 3 F_{\pi}^{2} / M_{\pi}^{2}-f_{\pi}^{2} / M_{\pi}^{2}-2 M_{d}^{2} / m_{d}^{2} \cdot f_{\pi}^{2} / M_{\pi}^{2}-y(y-1) n_{f} f_{\pi}^{4} / m_{d}^{2} f_{d}^{2}=0 .
\end{aligned}
$$

Physical parameters from dilaton EFT fits: The fitted posterior distributions of the physical parameters $f_{\pi}, B_{\pi}, y, m_{d} / f_{\pi}, f_{d} / f_{\pi}$ and their correlations are shown in Fig. 4 and Fig. 5. Approx- 


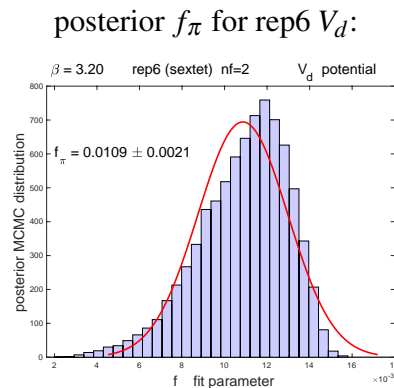

posterior $\gamma$ for rep6 $V_{d}$ :

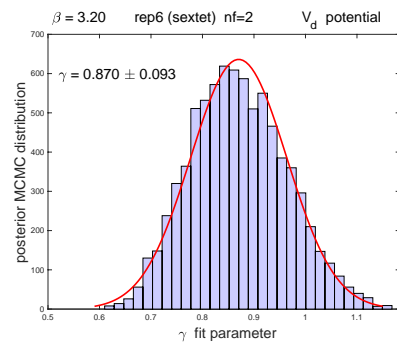

posterior $B_{\pi}$ for rep6 $V_{d}$ :

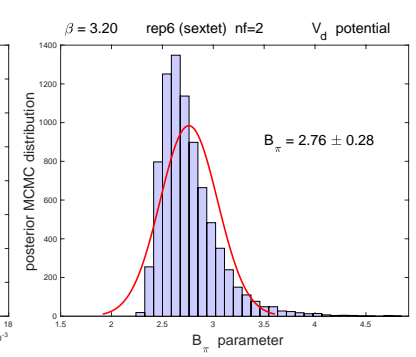

posterior $m_{d} / f_{\pi}$ for rep6 $V_{d}$ :

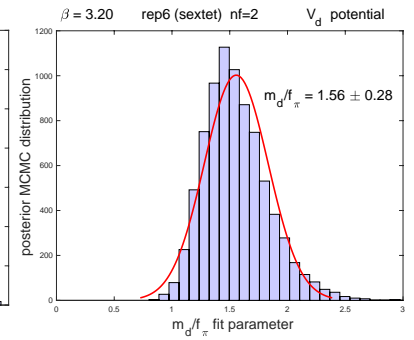

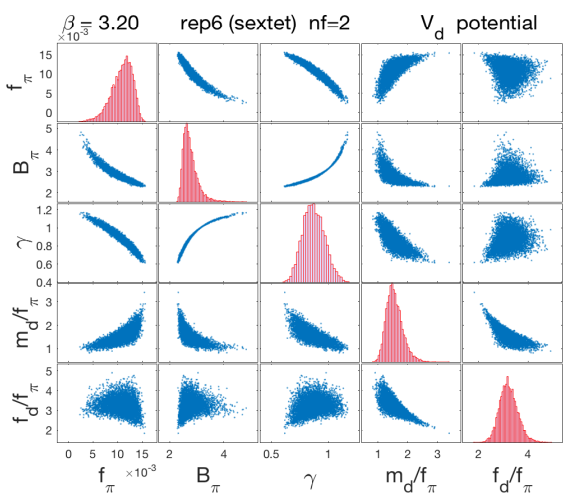

Matrix plot of the five fitted physical parameters with posterior histogram in the diagonal and off-diagonal scatter plots of the correlations. Four of the histograms are also shown on the left with fitted means and $1 \sigma$ equivalent percentile errors (the distributions are close to normal).

Figure 4: MCMC based posterior probability distributions of five fitted physical parameters and their correlations for the $V_{d}$ choice of the dilaton potential (rep6 sextet model). The MCMC algorithm is explained in the text. Red lines indicate fits of normal distributions to the histograms which show some deviations from the Gaussian shape as expected.

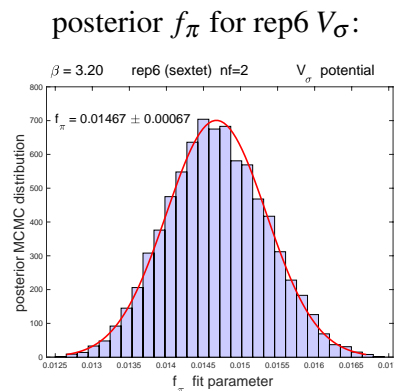

posterior $\gamma$ for rep6 $V_{\sigma}$ :

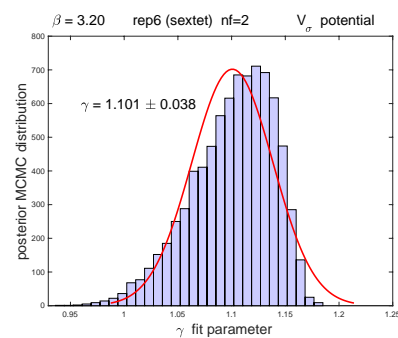

posterior $B_{\pi}$ for rep6 $V_{\sigma}$ :

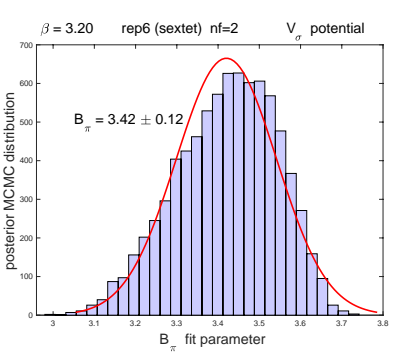

posterior $m_{d} / f_{\pi}$ for rep6 $V_{\sigma}$ :

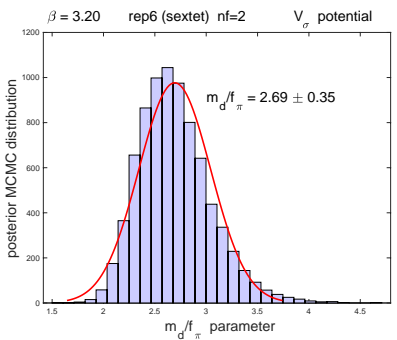

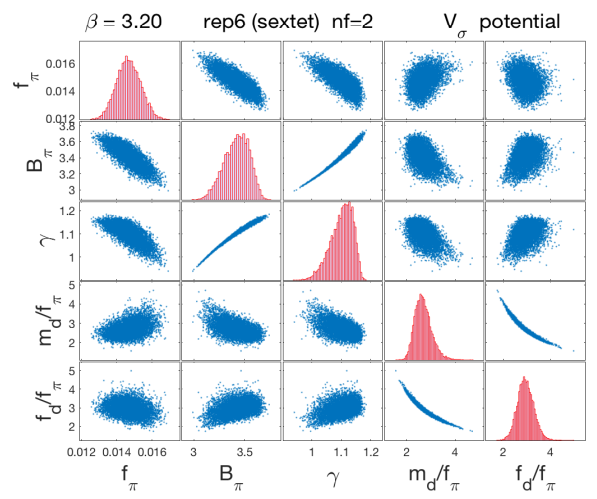

Matrix plot of the five fitted physical parameters with their posterior histograms in the diagonal and offdiagonal scatter plots of their correlations. Four of the histograms are also shown on the left with fitted means and $1 \sigma$ equivalent percentile errors (the distributions are close to normal).

Figure 5: MCMC based posterior probability distributions of the five fitted physical parameters and their correlations for the $V_{\sigma}$ choice of the dilaton potential (rep6 sextet model). Again, red lines indicate fits of normal distributions to the histograms which show some deviations from the Gaussian shape as expected.

imating the IML procedure, the distributions were generated in two stages. At the first stage, $M_{\pi}(m), F_{\pi}(m)$ correlated pairs of distribution functions were generated from extrapolation to infinite volume using finite size scaling (FSS) analysis separately at each fermion mass. Lattice input to the FSS fits was provided by three pairs of six $M_{\pi}\left(m, L, L_{t}\right), F_{\pi}\left(m, L, L_{t}\right)$ data from three volumes with linear sizes $L, L_{t}$ in respective spatial and time directions. Maximum Likelihood 
FSS fits gave us correlated infinite volume $M_{\pi}(m), F_{\pi}(m)$ pairs with covariance matrices which were used in Markov Chain Monte Carlo (MCMC) samples to generate the posterior probability distributions of the $M_{\pi}(m), F_{\pi}(m)$ pairs as input for the second stage of the fitting procedure. $M_{d}(m)$ probability distributions for input to the second stage were generated without FSS from normal distributions with previously determined variances at the largest available volume for each $m$. For each drawing from the $M_{\pi}(m), F_{\pi}(m), M_{d}(m)$ distributions, 12 input data were selected using $m=0.015 / 0.020 / 0.030 / 0.040$ for the non-linear fitting procedure of the five physical parameters from respective Eqs.(4.3-4.7) of the two dilaton potentials. We checked that adding the lowest fermion mass $m=0.0010$ with incomplete FSS for $F_{\pi}$ would not have any significant effect on the $M_{\pi}(m), F_{\pi}(m)$ distributions or the posterior distributions of the fitted physical parameters at the second stage. The posterior distributions of the five fitted physical parameters and their correlations were generated from the order of ten thousand drawings from the $M_{\pi}(m), F_{\pi}(m), M_{d}(m)$ distributions as shown in Fig. 4 and Fig. 5.

Results for the $V_{d}(\chi)$ choice of the dilaton potential in Eq.(4.2a) have some remarkable features with important implications for added post-conference analysis. The dilaton mass $m_{d} / f_{\pi}=$ $1.56(28)$ is dramatically lower than extrapolated results from chiral perturbation theory with less control. If confirmed, this light dilaton state alone could change our perspective on the sextet model for future investigations. The anomalous dimension $\gamma=0.870(93)$ is consistent with direct determination from the renormalized mode number distribution of the Dirac operator. The estimate of $f_{\pi}=0.0109(21)$ is lower than what was obtained in earlier $\chi P T$ fits which might require the recalibration of the separation between the $0^{++}$scalar and the associated heavy resonance spectrum. The value of $B_{\pi}=2.76(28)$ is close to what was determined from $\chi P T$ as shown in Fig. 3. However, the result $f_{d} / f_{\pi}=2.94$ (35) would present phenomenological difficulties for potential BSM applications.

Perhaps not surprisingly, results from the choice $V_{\sigma}(\chi)$ in Eq.(4.2b) are closer to what was expected from $\sigma$-model inspired $\chi P T$ estimates. The value of $B_{\pi}=3.42(12)$ is practically the same as what we obtained from chiral $\log$ fits in Section 3 and the dilaton mass is heavier in $f_{\pi}$ units, $m_{d} / f_{\pi}=2.69(35)$, closer to what we expected earlier for the $0^{++}$scalar. The ratio $f_{d} / f_{\pi}=3.22(37)$ is close to what we obtained with the choice of $V_{d}(\chi)$, with similar challenges for Electroweak embedding.

Sensitivity of the light scalar mass to the choice of the dilaton potential is the most striking outcome of the dilaton analysis in the sextet model. This might require revised strategies in future work including questions on the size of corrections to the tree-level approximation, affected by the small value of $f_{\pi}$. It is interesting to note that the $V_{d}(\chi)$ choice from Eq.(4.2a) is preferred near the $\mathrm{CW}$ from theoretical arguments in [1], not directly applicable to the sextet model.

\subsection{Dilaton EFT fits from $\mathbf{V}_{\sigma}$ and $\mathbf{V}_{d}$ dilaton potentials: $\mathbf{n}_{f}=8$ model}

With input data from [7], the histograms of four fitted physical parameters $f_{\pi}, B_{\pi}, y, m_{d} / f_{\pi}$. $f_{d} / f_{\pi}$ and their correlations are shown in Fig. 7 and Fig. 6. Using the largest volumes from [7] did not allow us for MCMC based FSS, instead normal distributions of $M_{\pi}, F_{\pi}$ were inputs to the fitting procedure. After experimenting with five-parameter fits, we reduced the analysis to four physical parameters without $M_{d}$ input. Accordingly, we did not use Eq.(4.5) and (4.7) with inputs from $M_{d}$ in [7], and only the product $m_{d} / f_{\pi} \cdot f_{d} / f_{\pi}$ was fitted without separating $m_{d} / f_{\pi}$. 

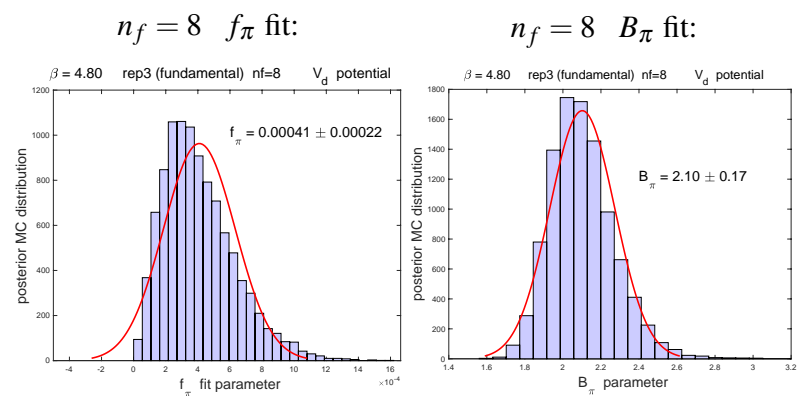

$n_{f}=8 \quad \gamma$ fit: $n_{f}=8 \quad m_{d} / f_{\pi} \cdot f_{d} / f_{\pi}$ fit:
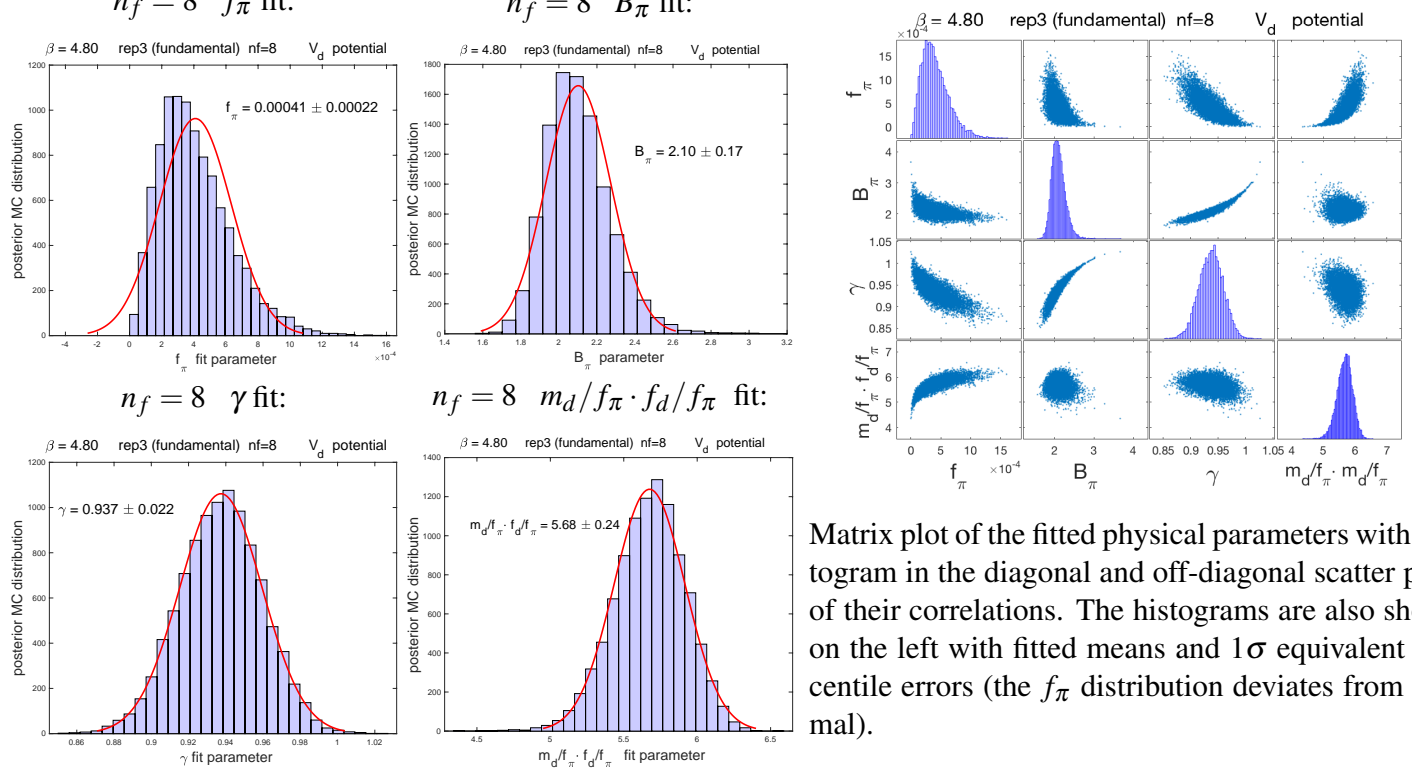

Matrix plot of the fitted physical parameters with histogram in the diagonal and off-diagonal scatter plots of their correlations. The histograms are also shown on the left with fitted means and $1 \sigma$ equivalent percentile errors (the $f_{\pi}$ distribution deviates from normal).

Figure 6: Histograms of the four fitted physical parameters and scatter plots of their correlations for the $V_{d}$ choice of the dilaton potential (rep3 $n_{f}=8$ model).

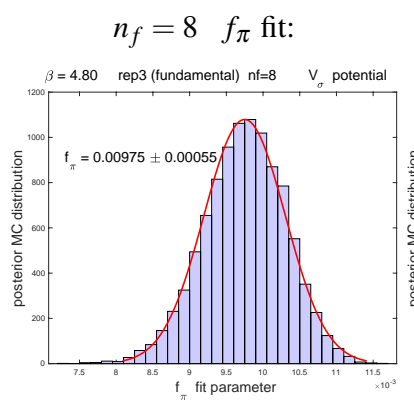

$n_{f}=8 \quad \gamma$ fit:

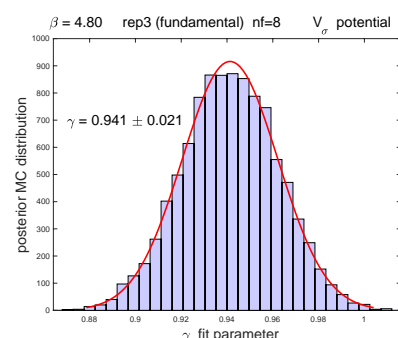

Figure 7: Histograms of four fitted physical parameters and scatter plots of their correlations for the $V_{\sigma}$ choice of the dilaton potential (rep3 $n_{f}=8$ model).

The $V_{\sigma}$ based $n_{f}=8$ fits do not show unexpected features. On the other hand, we had difficulties to interpret the $V_{d}$ based dilaton fits, in particular the very low value of $f_{\pi}=0.00041$ (22) calling into question the leading tree-level approximation to $V_{d}$ based tests of the dilaton EFT. We will return to investigate some stable way of including the scalar mass $M_{d}$ directly in the fitting procedure.

There is, however, an important aspect of dilaton EFT based fits to the $n_{f}=8$ model which is 
not affected by details of our fitting procedure. It was argued in [30] and presented at the conference [31] that the analysis of the $n_{f}=8$ model in $[12,13]$ is based on input data in the high-mass range of fermion mass deformations where $M_{\pi}$ and $F_{\pi}$ would show conformal scaling. It was estimated in $[30,31]$ that two orders of magnitude drop would be required from the currently available fermion mass range before the onset of chiral behavior is reached at very low fermion masses, outside the reach of realistic lattice simulation. The argument was based on Eq.(4.1) of the EFT for the choice $V_{d}$ of the dilaton potential in Eq.(4.2a). We checked our input data set $M_{\pi}, F_{\pi}$ from the
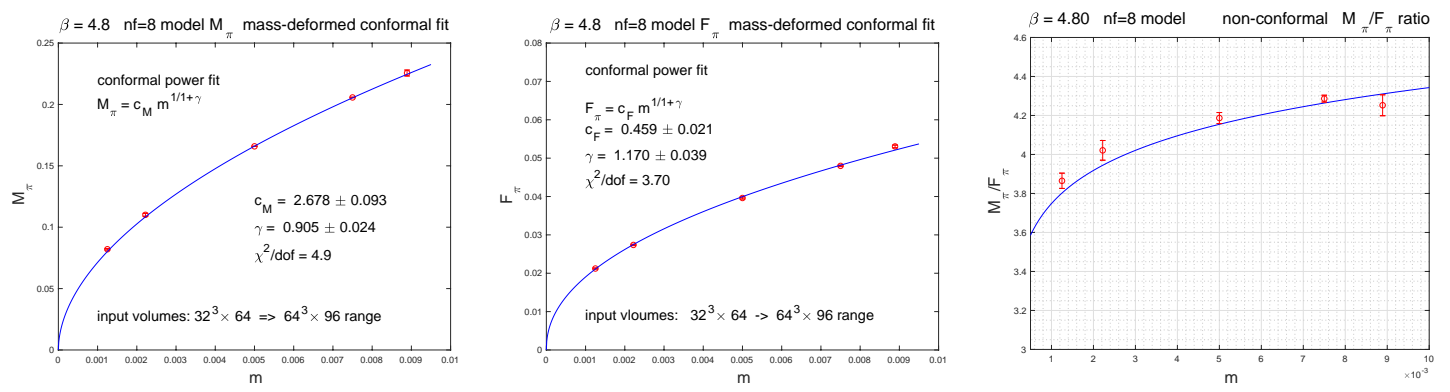

Figure 8: Inconsistencies of the conformal tests in the $n_{f}=8$ model, exhibiting considerable variation of the $M_{\pi} / F_{\pi}$ ratio in the fitted fermion mass range from incompatibility conformal exponents between $M_{\pi}$ and $F_{\pi}$.

recent LSD publication [7] for conformal behavior in the fermion mass range of the input data. The results in Fig. 8 for $M_{\pi}$ and $F_{\pi}$ do not show conformal behavior in the mass range of the dilaton analysis with considerable variation of the $M_{\pi} / F_{\pi}$ ratio and inconsistent conformal $\gamma$ exponents from forced conformal fits of $M_{\pi}$ and $F_{\pi}$. Although the identification of current $n_{f}=8$ simulations with the high-mass conformal regime will require correction terms [31], the estimated two order of magnitude drop in $m$ for reaching the regime of $\chi S B$ might not be far from what is required. At the conference we presented an idea how this large drop in $m$ might be reached instead in the $\varepsilon$-regime of $\chi S B$.

\section{Dilaton EFT analysis in the $\varepsilon$-regime and RMT}

In the $\varepsilon$-regime, close to the chiral limit where the pion correlation length far exceeds the linear size of the finite volume, the EFT Lagrangian of Eq.(4.1) simplifies to

$$
\mathscr{L}_{\varepsilon}=\frac{1}{2} \partial_{\mu} \chi \partial_{\mu} \chi-V_{d}(\chi)+\frac{m_{\pi}^{2} f_{\pi}^{2}}{4}\left(\frac{\chi}{f_{d}}\right)^{y} \operatorname{tr}\left[\Sigma_{0}+\Sigma_{0}^{\dagger}\right] .
$$

In Eq.(5.1) the coupling of the dilaton to the $\Sigma_{0}$ zero mode of the pion field is represented by the $\chi(x)$ field and can be treated by systematic expansion. In the strict $m \rightarrow 0$ chiral limit the pions become decoupled from the dilaton field. The challenge of this approach is to get close enough to the chiral limit at extremely small fermion masses. In fact, from careful studies of the lowest eigenvalues of the Dirac operator we determined that this limit would be feasible in large volume simulations at extremely small $m$ values. The feasibility was demonstrated by decreasing the fermion mass $m$ two orders of magnitude, down to $m=0.000010$ at the sextet gauge coupling $\beta=3.20$ with an estimated inverse pion mass of $M_{\pi}^{-1} \approx 125$ in the equivalent infinite volume p-regime. The simulation results from $64^{4}$ and $48^{3} \times 96$ lattice volumes with $M_{\pi} \cdot L<1$ at $m=$ 0.000010 are shown in Fig. 9. 

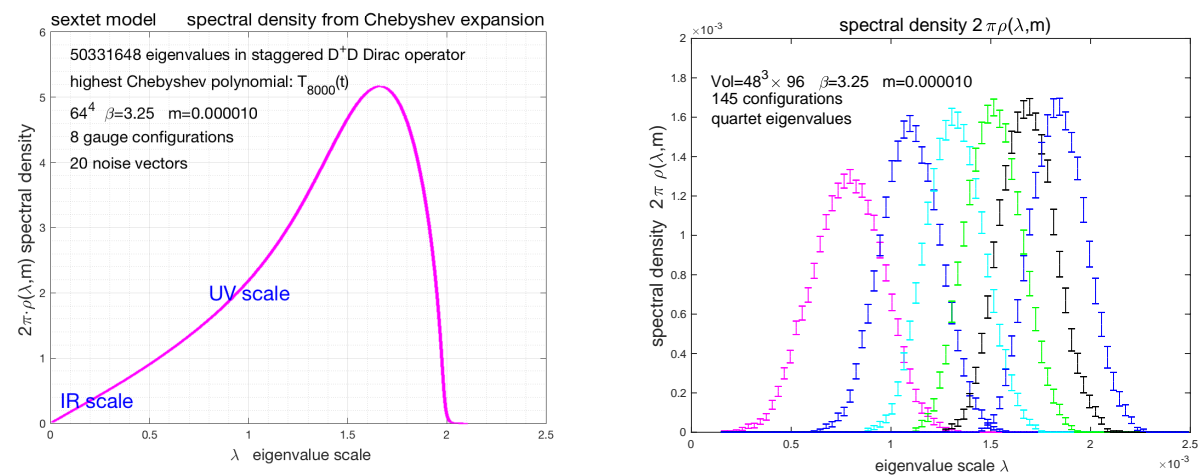

Figure 9: The spectral density of the full spectrum in the sextet model is shown on the left panel. Quartet averages of the lowest 24 eigenvalues are shown on the right panel for input into RMT analysis of the $\varepsilon$-regime. The shape and distribution of the lowest eigenvalues favors $\chi S B$ close to the chiral limit.

In the limit of asymmetric aspect ratio $L / L_{t} \rightarrow 0$ at fixed spatial size $L$ we cross over to the $\delta$-regime where in the chiral limit the dilaton EFT is further simplified for rotator analysis of the pion and the (de)coupling of the dilaton field,

$$
\mathscr{L}_{\delta}=\frac{1}{2} \partial_{\mu} \chi \partial_{\mu} \chi-V(\chi)+\frac{f_{\pi}^{2}}{4}\left(\frac{\chi}{f_{d}}\right)^{2} \operatorname{tr}\left[\partial_{t} \Sigma_{0} \partial_{t} \Sigma_{0}^{\dagger}\right] .
$$

The time derivative $\partial_{t} \Sigma_{0}$ of the zero three-momentum component of the pion field controls the coupled rotator dynamics on the SU(2) group manifold. Further analysis of Eqs. $(5.1,5.2)$ remains outside the scope of this report.

\section{Conclusions}

Based on the hypothesis of dilaton EFT description, tantalizing test results were obtained from the analysis of the sextet model with particularly interesting physical parameters for the $V_{d}$ form of the dilaton potential. It is important to note that the dilaton description of the light scalar from broken scale invariance does not follow from the $\beta$-function based walking behavior. Conformal symmetry breaking is not necessarily coupled to walking and requires better theoretical understanding. In addition, extended statistical analysis will be required for the full implementation of the implicit Maximum Likelihood method to assess the sensitivity and quality of our fitting procedure to different forms of the dilaton potential. Ratios of physical parameters depend on the $V_{d}$ or $V_{\sigma}$ form of the dilaton potential at fixed lattice spacing, calling for precision studies when the cutoff is varied in large volumes and close to the chiral limit. The $\varepsilon$-regime offers new opportunities, perhaps with direct determination of the effective dilaton potential from methods we developed and tested earlier in Yukawa theories of scalar fields and fermions [43,44]. It is also an important open question, if the application of the dilaton EFT survives other tests of fermion mass deformations, like $\chi P T$ effects in the chiral condensate, or the renormalized gauge coupling on the gradient flow.

\section{Acknowledgments}

We acknowledge support by the DOE under grant DE-SC0009919, by the NSF under grant 1620845, and by the Deutsche Forschungsgemeinschaft grant SFB-TR 55. Computational resources were provided by the DOE INCITE program on the ALCF BG/Q platform, by USQCD at Fermilab, by the University of Wuppertal, and by the Juelich Supercomputing Center on Juqueen. 


\section{References}

[1] M. Golterman and Y. Shamir, Low-energy effective action for pions and a dilatonic meson, Phys. Rev. D94 (2016) 054502 [1603.04575].

[2] Z. Fodor, K. Holland, J. Kuti, D. Nogradi and C. H. Wong, Can a light Higgs impostor hide in composite gauge models?, PoS LATTICE2013 (2014) 062.

[3] J. Kuti, The Higgs particle and the lattice, PoS LATTICE2013 (2014) 004.

[4] Z. Fodor, K. Holland, J. Kuti, S. Mondal, D. Nogradi and C. H. Wong, Status of a minimal composite Higgs theory, PoS LATTICE2015 (2016) 219 [1605. 08750].

[5] Z. Fodor, K. Holland, J. Kuti, S. Mondal, D. Nogradi and C. H. Wong, Fate of the conformal fixed point with twelve massless fermions and SU(3) gauge group, Phys. Rev. D94 (2016) 091501.

[6] Z. Fodor, K. Holland, J. Kuti, D. Nogradi and C. H. Wong, The twelve-flavor $\beta$-function and dilaton tests of the sextet scalar, EPJ Web Conf. 175 (2018) 08015 [1712.08594].

[7] LAtTice Strong Dynamics collaboration, T. Appelquist et al., Nonperturbative investigations of SU(3) gauge theory with eight dynamical flavors, 1807.08411.

[8] Z. Fodor, K. Holland, J. Kuti, D. Nogradi and C. H. Wong, The Yang-Mills gradient flow in finite volume, JHEP 11 (2012) 007 [1208.1051].

[9] Z. Fodor, K. Holland, J. Kuti, S. Mondal, D. Nogradi and C. H. Wong, The running coupling of 8 flavors and 3 colors, JHEP 06 (2015) 019 [1503.01132].

[10] LATKMI collaboration, Y. Aoki et al., Light composite scalar in eight-flavor QCD on the lattice, Phys. Rev. D89 (2014) 111502.

[11] T. Appelquist et al., Strongly interacting dynamics and the search for new physics at the LHC, 1601.04027.

[12] T. Appelquist, J. Ingoldby and M. Piai, Dilaton EFT Framework For Lattice Data, JHEP 07 (2017) $035[1702.04410]$.

[13] T. Appelquist, J. Ingoldby and M. Piai, Analysis of a Dilaton EFT for Lattice Data, JHEP 03 (2018) 039 [1711.00067].

[14] Z. Fodor, K. Holland, J. Kuti, D. Nogradi and C. H. Wong, Extended investigation of the twelve-flavor $\beta$-function, Phys. Lett. B779 (2018) 230.

[15] D. Nogradi, “Talk, presented at Lattice 2018, July 22-28, 2018, East Lansing, Michigan.”

[16] T.-W. Chiu, Discrete $\beta$-function of the SU(3) gauge theory with 10 massless domain-wall fermions, PoS LATTICE2016 (2017) 228.

[17] T.-W. Chiu, Improved study of the $\beta$-function of $S U(3)$ gauge theory with $N_{f}=10$ massless domain-wall/overlap fermions, 1811.01729.

[18] A. Cheng, A. Hasenfratz, Y. Liu, G. Petropoulos and D. Schaich, Improving the continuum limit of gradient flow step scaling, JHEP 05 (2014) 137 [1404.0984].

[19] A. Hasenfratz and D. Schaich, Nonperturbative $\beta$ function of twelve-flavor SU(3) gauge theory, JHEP 02 (2018) 132 [1610.10004].

[20] A. Hasenfratz, C. Rebbi and O. Witzel, Determination of the $N_{f}=12$ step scaling function using Möbius domain wall fermions, in Lattice 2018, 2018, 1810.05176. 
[21] Z. Fodor, K. Holland, J. Kuti, S. Mondal, D. Nogradi and C. H. Wong, The running coupling of the minimal sextet composite Higgs model, JHEP 09 (2015) 039 [1506. 0659 9].

[22] Z. Fodor, K. Holland, J. Kuti, D. Nogradi and C. H. Wong, A new method for the beta function in the chiral symmetry broken phase, in Lattice 2017, 2017, 1711. 04833.

[23] P. A. Baikov, K. G. Chetyrkin and J. H. Kühn, Five-Loop Running of the QCD coupling constant, Phys. Rev. Lett. 118 (2017) 082002 [1606.08659].

[24] M. Hansen, V. Drach and C. Pica, SU(3) sextet model with Wilson fermions, Phys. Rev. D96 (2017) 034518 [1705.11010].

[25] J. Kuti, “Talk, presented at Lattice 2017, June 18-24, 2017, Granada, Spain .”

[26] J. Soto, P. Talavera and J. Tarrus, Chiral Effective Theory with A Light Scalar and Lattice QCD, Nucl. Phys. B866 (2013) 270 [1110.6156].

[27] M. Hansen, K. Langæble and F. Sannino, Extending Chiral Perturbation Theory with an Isosinglet Scalar, Phys. Rev. D95 (2017) 036005 [1610.02904].

[28] M. Golterman and Y. Shamir, Effective pion mass term and the trace anomaly, Phys. Rev. D95 (2017) 016003 [1611.04275].

[29] W. D. Goldberger, B. Grinstein and W. Skiba, Distinguishing the Higgs boson from the dilaton at the Large Hadron Collider, Phys. Rev. Lett. 100 (2008) 111802 [0708 . 1463 ].

[30] M. Golterman and Y. Shamir, The large-mass regime of the dilaton-pion low-energy effective theory, Phys. Rev. D98 (2018) 056025 [1805.00198].

[31] M. Golterman and Y. Shamir, The large-mass regime of confining but nearly conformal gauge theories, in Lattice 2018, 2018, 1810 . 05353.

[32] A. A. Migdal and M. A. Shifman, Dilaton Effective Lagrangian in Gluodynamics, Phys. Lett. 114B (1982) 445.

[33] J. R. Ellis and J. Lanik, IS SCALAR GLUONIUM OBSERVABLE?, Phys. Lett. 150B (1985) 289.

[34] W. A. Bardeen, C. N. Leung and S. T. Love, The Dilaton and Chiral Symmetry Breaking, Phys. Rev. Lett. 56 (1986) 1230.

[35] C. N. Leung, S. T. Love and W. A. Bardeen, Aspects of Dynamical Symmetry Breaking in Gauge Field Theories, Nucl. Phys. B323 (1989) 493.

[36] J. F. Donoghue and H. Leutwyler, Energy and momentum in chiral theories, Z. Phys. C52 (1991) 343.

[37] Z. Chacko and R. K. Mishra, Effective Theory of a Light Dilaton, Phys. Rev. D87 (2013) 115006.

[38] L. Vecchi, Phenomenology of a light scalar: the dilaton, Phys. Rev. D82 (2010) 076009.

[39] S. Matsuzaki and K. Yamawaki, Dilaton Chiral Perturbation Theory: Determining the Mass and Decay Constant of the Technidilaton on the Lattice, Phys. Rev. Lett. 113 (2014) 082002.

[40] LATKMI collaboration, Y. Aoki et al., Light flavor-singlet scalars and walking signals in $N_{f}=8$ QCD on the lattice, Phys. Rev. D96 (2017) 014508 [1610.07011].

[41] R. J. Crewther and L. C. Tunstall, $\Delta I=1 / 2$ rule for kaon decays derived from $Q C D$ infrared fixed point, Phys. Rev. D91 (2015) 034016 [1312.3319].

[42] O. Catà, R. J. Crewther and L. C. Tunstall, Crawling technicolor, 1803.08513.

[43] Y. Shen, J. Kuti, L. Lin and P. Rossi, Lattice1988, pp. 99-104, 1988.

[44] Z. Fodor, K. Holland, J. Kuti, D. Nogradi and C. Schroeder, New Higgs physics from the lattice, PoS LATTICE2007 (2007) 056 [0710.3151]. 